NASA/TM-1999-209134

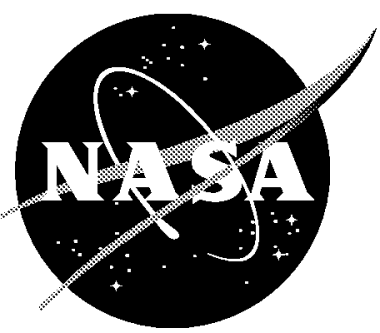

\title{
Towards a Rational Model for the Triple Velocity Correlations of Turbulence
}

B. A. Younis

City University, London, England

T. B. Gatski

Langley Research Center, Hampton, Virginia

C. G. Speziale

Boston University, Boston, Massachusetts 
The NASA STI Program Office ... in Profile

Since its founding, NASA has been dedicated to the advancement of aeronautics and space science. The NASA Scientific and Technical Information (STI) Program Office plays a key part in helping NASA maintain this important role.

The NASA STI Program Office is operated by Langley Research Center, the lead center for NASA's scientific and technical information. The NASA STI Program Office provides access to the NASA STI Database, the largest collection of aeronautical and space science STI in the world. The Program Office is also NASA's institutional mechanism for disseminating the results of its research and development activities. These results are published by NASA in the NASA STI Report Series, which includes the following report types:

- TECHNICAL PUBLICATION. Reports of completed research or a major significant phase of research that present the results of NASA programs and include extensive data or theoretical analysis. Includes compilations of significant scientific and technical data and information deemed to be of continuing reference value. NASA counterpart and peer-reviewed formal professional papers, but having less stringent limitations on manuscript length and extent of graphic presentations.

- TECHNICAL MEMORANDUM. Scientific and technical findings that are preliminary or of specialized interest, e.g., quick release reports, working papers, and bibliographies that contain minimal annotation. Does not contain extensive analysis.

- CONTRACTOR REPORT. Scientific and technical findings by NASA-sponsored contractors and grantees.
- CONFERENCE PUBLICATION. Collected papers from scientific and technical conferences, symposia, seminars, or other meetings sponsored or co-sponsored by NASA.

- SPECial PUBLiCATION. Scientific, technical, or historical information from NASA programs, projects, and missions, often concerned with subjects having substantial public interest.

- TECHNICAL TRANSLATION. Englishlanguage translations of foreign scientific and technical material pertinent to NASA's mission.

Specialized services that complement the STI Program Office's diverse offerings include creating custom thesauri, building customized databases, organizing and publishing research results... even providing videos.

For more information about the NASA STI Program Office, see the following:

- Access the NASA STI Program Home Page at http://www.sti.nasa.gov

- E-mail your question via the Internet to help@sti.nasa.gov

- Fax your question to the NASA STI Help Desk at (301) 621-0134

- Phone the NASA STI Help Desk at (301) $621-0390$

- Write to: NASA STI Help Desk NASA Center for AeroSpace Information 7121 Standard Drive Hanover, MD 21076-1320 
NASA/TM-1999-209134

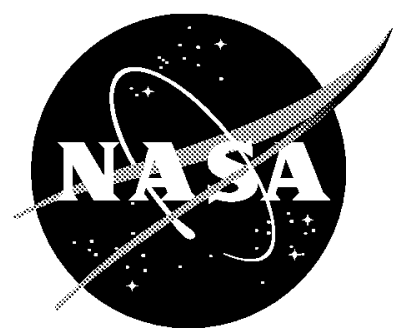

\section{Towards a Rational Model for the Triple Velocity Correlations of Turbulence}

B. A. Younis

City University, London, England

T. B. Gatski

Langley Research Center, Hampton, Virginia

C. G. Speziale

Boston University, Boston, Massachusetts

National Aeronautics and

Space Administration

Langley Research Center

Hampton, Virginia 23681-2199 
Available from:

NASA Center for AeroSpace Information (CASI) 7121 Standard Drive

Hanover, MD 21076-1320

(301) 621-0390
National Technical Information Service (NTIS) 5285 Port Royal Road

Springfield, VA 22161-2171

(703) 605-6000 


\title{
Towards a Rational Model for the Triple Velocity Correlations of Turbulence
}

\author{
B. A. Younis \\ Department of Civil Engineering \\ City University, London EC1V OHB, UK \\ T. B. Gatski \\ Aerodynamic and Acoustic Methods Branch \\ NASA Langley Research Center, Hampton, VA 23681, USA \\ C. G. Speziale \\ Aerospace and Mechanical Engineering Department \\ Boston University, Boston, MA 02015, USA
}

\begin{abstract}
This paper presents a rational approach to modelling the triple velocity correlations that appear in the transport equations for the Reynolds stresses. All existing models of these correlations have largely been formulated on phenomenological grounds and are defective in one important aspect: they all neglect to allow for the dependence of these correlations on the local gradients of mean velocity. The mathematical necessity for this dependence will be demonstrated in the paper. The present contribution lies in the novel use of Group Representation Theory to determine the most general tensorial form of these correlations in terms of all the second-and third-order tensor quantities that appear in the exact equations that govern their evolution. The requisite representation did not exist in the literature and therefore had to be developed specifically for this purpose by Professor G. F. Smith. The outcome of this work is a mathematical framework for the construction of algebraic, explicit, and rational models for the triple velocity correlations that are theoretically consistent and include all the correct dependencies. Previous models are reviewed, and all are shown to be an incomplete subset of this new representation, even to lowest order.
\end{abstract}

\section{Introduction}

The state of the art in closure of the Reynolds-averaged form of the Navier-Stokes equations requires the solution of a differential transport equation for each nonzero component of the Reynolds stress tensor $\left(\overline{u_{i} u_{j}}\right)$. These equations are exact but contain a number of correlations which are unknown and must first be modelled in terms of known or knowable quantities. One such correlation, and the focus of this work, is the time-averaged triple velocity product $\left(\overline{u_{i} u_{j} u_{k}}\right)$ whose spatial gradients represent the rate at which the Reynolds stresses are transported by the turbulent fluctuations. Conventional models of these correlations have been formulated with this physical role in mind. Specifically, and by analogy with the gradient transport hypothesis, Daly and Harlow (1970) assumed that the triple correlations are proportional to the spatial gradients of the Reynolds stresses to obtain

$$
-\overline{u_{i} u_{j} u_{k}}=C_{s} \frac{k}{\epsilon} \overline{u_{k} u_{l}} \frac{\partial \overline{u_{i} u_{j}}}{\partial x_{l}}
$$


where $k$ is the turbulence kinetic energy (i.e., half the trace of $\overline{u_{i} u_{i}}$ ), $\epsilon$ is its dissipation by viscous action, and $C_{s}$ is a proportionality coefficient. The Daly-Harlow model is the most widely used model in advanced simulations methods. However, while the triple velocity correlations themselves are independent of the order of the indices, the representation (the right-hand side of Equation 1) is not invariant under the permutation of the same indices. This is not usually a problem in thin shear layers where the flow direction is known a priori. It does, however, become so in general flows where turbulent mixing can be significant in all coordinate directions. Several alternative models have been reported in the literature, and discussion of some of these is deferred until Section III. Suffice it to say here that these models, while being considerably more complex than Daly and Harlow, have not produced tangible or consistent improvements in the quality of predictions. The lack of improvement is equally true in both simple, two-dimensional free shear flows as well as in rapidly evolving flows in the presence of complex strain fields. This is the case, in our opinion, because all proposed models fail to reflect the dependence of the triple velocity correlations on an essential mechanism for turbulence transport, namely the spatial gradients of the mean velocity. That a rational model for $\overline{u_{i} u_{j} u_{k}}$ should exhibit explicit dependence on the gradients of mean velocity will be demonstrated conclusively in the next section. The provision of a theoretical framework for the formulation of such a model and a demonstration of how this explicit dependence can be introduced without recourse to empiricism provide the principal motivation for the present work. In Section II, the mathematical basis of the problem is presented. Our proposals for the formulation of a rational model for the triple velocity correlation are put forward in Section III and are discussed there in relation to existing models. Conclusions are given in Section IV. In developing a rational model for the triple velocity correlations, we sought to find a general representation of a third-order tensor in terms of both second- and thirdorder tensors. No such representation could be found in the published literature, and the need therefore arose for the development of the mathematical representation to specifically meet this requirement. This challenging task was performed by Professor G. F. Smith; his original work, which will no doubt find application in other fields of science, is included in the appendix.

\section{The Mathematical Context}

We consider here the turbulent flow of a viscous, incompressible fluid with constant properties. The governing field equations are the Navier-Stokes and continuity equations, which are given by

$$
\begin{gathered}
\frac{D \tilde{u}_{i}}{D t}=-\frac{\partial \tilde{p}}{\partial x_{i}}+\nu \frac{\partial^{2} \tilde{u}_{i}}{\partial x_{j}^{2}}, \\
\frac{\partial \tilde{u}_{i}}{\partial x_{i}}=0,
\end{gathered}
$$

where $\tilde{u}_{i}$ is the instantaneous velocity vector, $\tilde{p}$ is the static pressure and $\nu$ is the kinematic viscosity of the fluid. In equations (2) and (3), the Einstein summation 
convention applies to repeated indices, and $D / D t$ is the convective derivative defined as

$$
\frac{D}{D t} \equiv \frac{\partial}{\partial t}+\tilde{u}_{j} \frac{\partial}{\partial x_{j}}
$$

The instantaneous velocity and pressure are decomposed into mean and fluctuating parts as follows:

$$
\tilde{u}_{i}=U_{i}+u_{i}, \quad \tilde{p}=P+p .
$$

By substituting (5) into equation (2) and applying the Reynolds averaging rules (cf. Hinze 1975), there results the Reynolds-averaged Navier-Stokes equation:

$$
\frac{D U_{i}}{D t}=-\frac{\partial P}{\partial x_{i}}+\nu \frac{\partial^{2} U_{i}}{\partial x_{j}^{2}}-\frac{\partial \overline{u_{i} u_{j}}}{\partial x_{j}}
$$

Due to the nonlinearity of the convection term in equation (2), the time-averaging operation introduces additional unknowns in the form of the Reynolds stress tensor $\overline{u_{i} u_{j}}$. This is the closure problem referred to in the Introduction. The most advanced methods for resolving this problem rely on the solution of differential transport equations for $\overline{u_{i} u_{j}}$. Derivation of these equations proceeds by multiplying the $i$-th component of equation (2) by $\tilde{u}_{j}$ and the $j$-th component by $\tilde{u}_{i}$. By summing and applying the Reynolds averaging rules, we have

$$
\begin{aligned}
\overbrace{\frac{D \overline{u_{i} u_{j}}}{D t}}^{\text {Convection }}= & -\overbrace{\left(\overline{u_{i} u_{k}} \frac{\partial U_{j}}{\partial x_{k}}+\overline{u_{j} u_{k}} \frac{\partial U_{i}}{\partial x_{k}}\right)}^{\text {Production }} \\
& -\overbrace{\frac{\partial}{\partial x_{k}}\left[\overline{u_{i} u_{j} u_{k}}+\frac{1}{\rho}\left(\overline{p^{\prime} u_{i}} \delta_{j k}+\overline{p^{\prime} u_{j}} \delta_{i k}\right)-\nu \frac{\partial u_{i} u_{j}}{\partial x_{k}}\right]}^{\text {Diffusion }} \\
& -\overbrace{2 \nu\left(\overline{\frac{\partial u_{i}}{\partial x_{k}} \frac{\partial u_{j}}{\partial x_{k}}}\right)}^{\text {Dissipation }}+\overbrace{\left.\frac{p^{\prime}\left(\frac{\partial u_{i}}{\partial x_{j}}+\frac{\partial u_{j}}{\partial x_{i}}\right)}{\rho(\text { Redistribution }}\right)}
\end{aligned}
$$

The Reynolds averaging process has clearly resulted in additional unknown turbulence correlations, another manifestation of the closure problem. The literature contains numerous proposals for approximating these correlations (Speziale 1991 provides a comprehensive review of the subject). Our interest here is in the triple fluctuatingvelocity components $\overline{u_{i} u_{j} u_{k}}$. The exact equation governing the evolution of $\overline{u_{i} u_{j} u_{k}}$ reads (cf. Chou 1945):

$$
\frac{D \overline{u_{i} u_{j} u_{k}}}{D t}=-\overbrace{\left(\overline{u_{i} u_{j} u_{l}} \frac{\partial U_{k}}{\partial x_{l}}+\overline{u_{j} u_{k} u_{l}} \frac{\partial U_{i}}{\partial x_{l}}+\overline{u_{k} u_{i} u_{l}} \frac{\partial U_{j}}{\partial x_{l}}\right)}^{\mathbf{I}}
$$




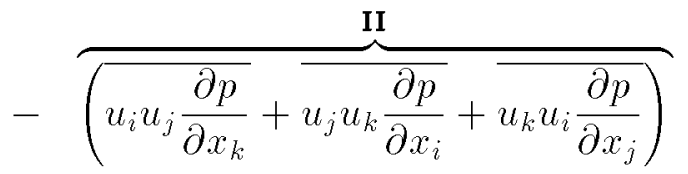

$$
\begin{aligned}
& +\overbrace{\left(\overline{u_{i} u_{j}} \frac{\partial \overline{u_{k} u_{l}}}{\partial x_{l}}+\overline{u_{j} u_{k}} \frac{\partial \overline{u_{i} u_{l}}}{\partial x_{l}}+\overline{u_{k} u_{i}} \frac{\partial \overline{u_{j} u_{l}}}{\partial x_{l}}\right)}^{\text {III }} \\
& -\overbrace{\frac{\partial}{\partial x_{l}} \overline{u_{i} u_{j} u_{k} u_{l}}}^{\mathbf{I V}}+\overbrace{\nu \frac{\partial^{2} \overline{u_{i} u_{j} u_{k}}}{\partial x_{l}^{2}}}^{\mathbf{v}}
\end{aligned}
$$

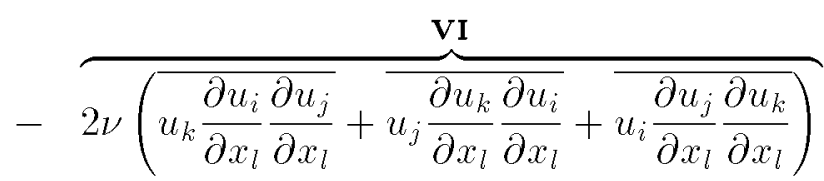

It is immediately clear from line $\mathbf{I}$ of this equation that the gradients of mean velocity appear explicitly in the exact equation for the triple velocity correlations. Further, and as was shown by Chou (1945), the gradients of mean velocity must also appear explicitly in the correlation between the Reynolds stresses and the pressure fluctuations (line II above). Thus, by differentiating equation (2) to obtain a Poisson equation for the instantaneous pressure, and then by subtracting the mean, Chou obtained an equation for the fluctuating pressure:

$$
\frac{1}{\rho} \frac{\partial^{2} p}{\partial x_{m}^{2}}=-\left[\frac{\partial^{2}\left(u_{m} u_{k}-\overline{u_{m} u_{k}}\right)}{\partial x_{k} \partial x_{m}}+2 \frac{\partial U_{m}}{\partial x_{k}} \frac{\partial u_{k}}{\partial x_{m}}\right]
$$

Then, with the assumption of homogeneous turbulence, and for regions of the flow remote from solid boundaries, the following approximate solution was obtained:

$$
\frac{1}{\rho}\left(\overline{u_{i} u_{j} \frac{\partial p}{\partial x_{k}}}+\overline{u_{j} u_{k} \frac{\partial p}{\partial x_{i}}}+\overline{u_{k} u_{i} \frac{\partial p}{\partial x_{j}}}\right)=b_{n m i j k} \frac{\partial U_{m}}{\partial x_{n}}+c_{i j k}
$$

The forms of the tensors, $b_{n m i j k}$ and $c_{i j k}$, that appear in equation (10) are not material for the present purposes (they are given by equation (5.8) of the original reference). What is important here is the presence of the mean velocity gradients in the solution. It therefore follows that a rational model for the triple velocity correlation must contain an explicit dependence on the gradients of mean velocity so that it remains consistent with the exact equation, and so that it stands a chance of representing the correct physics. Mathematically, this proposition implies that a model for the triple velocity correlations must be of the following functional form:

$$
\overline{u_{i} u_{j} u_{k}}=F_{i j k}\left(\overline{u_{l} u_{m}}, \epsilon, \frac{\partial \overline{u_{l} u_{m}}}{\partial x_{n}}, S_{m n}, W_{m n}\right)
$$

where $S_{m n}$ and $W_{m n}$ are, respectively, the mean rate of strain and the mean vorticity tensors:

$$
S_{m n}=\frac{1}{2}\left(\frac{\partial U_{m}}{\partial x_{n}}+\frac{\partial U_{n}}{\partial x_{m}}\right)
$$




$$
W_{m n}=\frac{1}{2}\left(\frac{\partial U_{m}}{\partial x_{n}}-\frac{\partial U_{n}}{\partial x_{m}}\right)
$$

The dissipation rate $\epsilon$ is included in the argument of the general functional representation in order to build up the turbulence length and time scales ( $l$ and $\tau$, respectively) from the relations:

$$
l \propto \frac{k^{3 / 2}}{\epsilon}, \quad \tau \propto \frac{k}{\epsilon}
$$

Inspection of the Daly and Harlow (1970) model, equation (1), shows that it follows the incomplete representation

$$
\overline{u_{i} u_{j} u_{k}}=F_{i j k}^{\prime}\left(\overline{u_{l} u_{m}}, \epsilon, \frac{\partial \overline{u_{l} u_{m}}}{\partial x_{n}}\right)
$$

This is also true of all the models that have appeared in the literature. Hanjalic and Launder (1972), for example, derived a model from simplification of equation (8), but although they recognized that a complete model for II should contain the gradients of mean velocity, they proceeded to neglect this contribution from their workings. Thus, while their model represents an improvement over that of equation (1) (in being symmetric under the permutation of indices), its use in actual flow simulations has not produced tangible improvements. A discussion of some of the published proposals for the triple velocity correlations is included in the next section. Suffice it to say that all turn out to be of the functional form given by equation (15) rather than the mathematically consistent form of equation (11). In the next secion, we put forward our proposals for the formulation of a rational and consistent alternative.

\section{The Present Proposal}

One way to proceed in the formulation of a rational model for $\overline{u_{i} u_{j} u_{k}}$ is to attempt a term-by-term approximation of the various unknown turbulence correlations that appear in equation (8). The obvious difficulty with this approach lies in formulating adequate approximations to the large number of additional unknown terms that are involved. An example is the term that involves the gradients of the quadruple velocity

correlation. An exact equation for this term is again not difficult to construct but will involve yet higher-order unknowns that will need to be approximated. Truncation of the process of introducing increasingly higher-order correlations can only be achieved by arbitrary considerations - something that is not in keeping with the present requirement for a rational formulation.

Instead, the present proposal is to use Group Representation Theory to construct, in accordance with the functional relation of equation (11), the most general algebraic representation for the symmetric third-order tensor $\overline{u_{i} u_{j} u_{k}}$ in terms of both secondand third-order tensors. Both symmetric and antisymmetric second-order tensors are to be included in the representation. This representation is also required to be invariant under the three-dimensional full orthogonal group $O_{3}$. 
Surprisingly, the required mathematical representation does not exist in the literature. Pennisi (1992) proposed a tensorial representation for a functional of a third-order tensor whose dependent variables consist of first- and second-order tensors. This representation. is clearly inadequate for the present purposes as it would lead to a model for the triple velocity correlations that does not contain dependence on the gradients of the Reynolds stresses. This dependence is required by term II in equation (11).

The necessary representation therefore had to be developed specifically for this work. This task was achieved by Professor G F Smith. As it forms a distinct contribution to the subject, details of the theory are reproduced fully in the appendix. The complete representation for the triple velocity correlations that is consistent with the functional of equation (11) admits tensor dependencies up to fourth order. We shall henceforth dismiss terms that are of orders three and four because of the large numbers involved, equation (A4), and more importantly, because these terms do not introduce tensor parameters that are not already present in the linear and bilinear representation.

With this simplification, the general representation of the triple velocity correlations suggested by Smith's theory consists of the following group of terms:

Group I: Terms that are linear in $\left(\overline{u_{i} u_{j}}\right)_{, k}$. There are three linearly independent terms of this type, as indicated by (A11).

Group II: Terms that are bilinear in $\left(\overline{u_{i} u_{j}}\right)_{, k}$ and $\overline{u_{i} u_{j}}$. There are eleven such terms (A12).

Group III: Terms that are bilinear in $\left(\overline{u_{i} u_{j}}\right)_{, k}$ and $S_{i j}$. There are eleven linearly independent terms of this type (A13).

Group IV: Terms that are bilinear in $\left(\overline{u_{i} u_{j}}\right)_{, k}$ and $W_{i j}$. There are five such terms (A14). There are thus thirty terms in all, the combination of which produces a general representation of the form

$$
\overline{u_{i} u_{j} u_{k}}=\sum_{\gamma=1}^{30} c_{\gamma} \Phi_{i j k}^{(\gamma)}
$$

where the $\Phi_{i j k}^{(\gamma)}(\gamma=1, \ldots, 30)$ are the third-order symmetric tensor-valued isotropic functions listed in A11-A14. The $c_{\gamma}$ are material constants.

We shall now consider the relation between this general representation and various ad-hoc models for the triple correlations. The literature contains a diverse assortment of such models, but these fall into two categories which are either linear or bilinear in the tensor dependencies shown in equation (15).

First we consider models that are linear in the Reynolds stresses $\overline{u_{i} u_{j}}$. Examples of these models are the proposals of Mellor and Herring (1973) (henceforth referred to as $M H$ ) and Shir (1973). The $M H$ model is given by

$$
-\overline{u_{i} u_{j} u_{k}}=C_{M H} \frac{k^{2}}{\epsilon}\left(\frac{\partial \overline{u_{j} u_{k}}}{\partial x_{i}}+\frac{\partial \overline{u_{k} u_{i}}}{\partial x_{j}}+\frac{\partial \overline{u_{i} u_{j}}}{\partial x_{k}}\right),
$$

where $C_{M H}$ is a constant. Shir's model contains only the third term in equation (17) and therefore does not exhibit correct symmetry. Both models are a sub-set of the 
general representation in equation (16). The $M H$ model, for example, corresponds to the $\sum A_{i j k}$ term of equation (A11). If all linear terms were to be included in a model, then two additional terms would appear, namely $A_{i p p} \delta_{j k}$ and $\sum A_{p p i} \delta_{j k}$. A consistent linear model for the triple velocity correlations would thus read

$$
\begin{aligned}
-\overline{u_{i} u_{j} u_{k}} & =C_{M H, 1} \frac{k^{2}}{\epsilon} \overbrace{\left(\frac{\partial \overline{u_{j} u_{k}}}{\partial x_{i}}+\frac{\partial \overline{u_{k} u_{i}}}{\partial x_{j}}+\frac{\partial \overline{u_{i} u_{j}}}{\partial x_{k}}\right)}^{\text {Mellor and Herring }} \\
& +C_{M H, 2} \frac{k^{2}}{\epsilon}\left(\frac{\partial \overline{u_{i} u_{p}}}{\partial x_{p}} \delta_{j k}+\frac{\partial \overline{u_{j} u_{p}}}{\partial x_{p}} \delta_{k i}+\frac{\partial \overline{u_{k} u_{p}}}{\partial x_{p}} \delta_{i j}\right) \\
& +C_{M H, 3} \frac{k^{2}}{\epsilon}\left(\frac{\partial k}{\partial x_{i}} \delta_{j k}+\frac{\partial k}{\partial x_{j}} \delta_{k i}+\frac{\partial k}{\partial x_{k}} \delta_{i j}\right)
\end{aligned}
$$

Incidentally, this more complete formulation was also considered by Mellor and Herring (1973) who discarded it on the grounds of computational expediency.

Next, we consider models that are bilinear in the tensor quantities. The Daly and Harlow (1970) model, equation (1), is of this category but, as already mentioned in the Introduction, is not independent of the order of indices and cannot therefore be included in the tensor representation shown in the appendix.

A more complicated, but tensorially admissible expression has been proposed by Hanjalic and Launder (1972) (hereafter $H L$ ). They arrived at their model by combining lines III and IV of equation (8) after a proposition by Millionshtchikov (1941) and by neglecting lines I and II entirely. In addition, the transport term on the left-hand side of the same equation to obtain the following algebraic representation:

$$
-\overline{u_{i} u_{j} u_{k}}=C_{H L} \frac{k}{\epsilon}\left(\overline{u_{i} u_{l}} \frac{\partial \overline{u_{j} u_{k}}}{\partial x_{l}}+\overline{u_{j} u_{l}} \frac{\partial \overline{u_{k} u_{i}}}{\partial x_{l}}+\overline{u_{k} u_{l}} \frac{\partial \overline{u_{i} u_{j}}}{\partial x_{l}}\right)
$$

where $C_{H L}$ is a constant. This model corresponds to the $\sum A_{i j p} \tau_{p k}$ term given in (A12). Note that the $M H$ model can be viewed as an isotropized version of the $H L$ model.

Another model which is bilinear in the tensor quantities is that of Lumley (1978). He considered weakly anisotropic and inhomogeneous flows and applied stochastic considerations to obtain

$$
-\overline{u_{i} u_{j} u_{k}}=C_{L}^{1} \frac{k}{\epsilon}\left[\mathcal{G}_{i j k}+C_{L}^{2}\left(\mathcal{G}_{i m m} \delta_{j k}+\mathcal{G}_{m j m} \delta_{i k}+\mathcal{G}_{m m k} \delta_{i j}\right)\right]
$$

In terms of the notation used here, $\mathcal{G}_{i j k}=\sum A_{i j p} \tau_{p k}$, and the model given in equation (20) can be rewritten as

$$
-\overline{u_{i} u_{j} u_{k}}=C_{L}^{1} \frac{k}{\epsilon}\left[\sum A_{i j p} \tau_{p k}+C_{L}^{2} \sum A_{i p q} \tau_{p q} \delta_{j k}\right]
$$

Equation (21) shows that Lumley's model is tensorially equivalent to that of Hanjalic and Launder in being bilinear in the tensor product of $\overline{u_{l} u_{m}}$ and $\partial \overline{u_{l} u_{m}} / \partial x_{n}$. Both models, however, are only a subset of the 11 possible bilinear basis tensors (see (A12)). 
Finally, Cormack et al. (1978) formulated a model in terms of the deviatoric part of the Reynolds stress tensor, $\overline{u_{i} u_{j}}-2 / 3 \delta_{i j} k$. In the mathematical framework outlined here, their model reads

$$
\begin{aligned}
-\overline{u_{i} u_{j} u_{k}} & =\frac{k^{2}}{\epsilon}\left(C_{C L S}^{1} \sum A_{i j k}+C_{C L S}^{2} \sum A_{p p i} \delta_{j k}+C_{C L S}^{3} A_{i p p} \delta_{j k}\right) \\
& +\frac{k}{\epsilon}\left(C_{C L S}^{4} \sum A_{i p p} \tau_{j k}+C_{C L S}^{5} \sum A_{p p i} \tau_{j k}+C_{C L S}^{6} \sum A_{p p q} \tau_{q i} \delta_{j k}\right)
\end{aligned}
$$

These six closure constants are linear combinations of the original four constants proposed by Cormack et al. (1978). Magnaudet (1993) also considered the Cormack model and provided a cross-diffusion extension which included the gradient of the isotropic dissipation rate. These terms were not included in the functional form proposed here, equation (11), as there is no evidence from equation (8) that such terms should be present.

It should be recognized that since the tensor representations given in (A11) - (A14) do not imply any asymptotic ordering of the terms, all these models should be viewed as arbitrary truncations of the full representation basis. Table I gives a summary of the models discussed and the corresponding basis terms involved. It is clear from Table I that the majority of these models are bilinear in the Reynolds stresses and their gradients. None of them contain the dependence on the mean velocity gradients that is required by the exact equation. It is not surprising, therefore, that there is little real

\begin{tabular}{|c|c|c|c|c|c|}
\hline \multirow[b]{2}{*}{$\begin{array}{l}\text { Basis } \\
\text { Tensor }\end{array}$} & \multicolumn{5}{|c|}{ Model } \\
\hline & $\begin{array}{l}\text { Hanjalic and Launder } \\
(1972)\end{array}$ & $\begin{array}{c}\text { Mellor and Herring } \\
(1973)\end{array}$ & $\begin{array}{r}\text { Lumley } \\
(1978)\end{array}$ & $\begin{array}{c}\text { Cormack et al } \\
(1978)\end{array}$ & $\begin{array}{l}\text { Magnaudet } \\
\text { (1993) }\end{array}$ \\
\hline$\left\{\begin{array}{l}A_{i j k} \\
A_{i p p} \delta_{j k} \\
A_{p p i} \delta_{j k}\end{array}\right.$ & & 0.073 & & $\begin{array}{r}0.069 \\
-0.136 \\
-0.632\end{array}$ & \\
\hline$\sum A_{i p p} \tau_{j k}$ & & & & 0.102 & 0.16 \\
\hline$\sum A_{i j p} \tau_{p k}$ & 0.11 & & $\begin{array}{l}0.098 \\
0.013\end{array}$ & -0.000 & 0.125 \\
\hline$\sum A_{p p q \tau q i} \delta_{j k}$ & & & & 0.192 & \\
\hline
\end{tabular}

Table I. Summary of $\overline{u_{i} u_{j} u_{k}}$ models and corresponding tensor bases

difference in their performance across a wide range of benchmark turbulent shear flows (see, e.g., Schwarz and Bradshaw 1994, and Cormack et al. 1978). There are a number of flows in which the ratio of turbulence kinetic energy production to dissipation rates is near unity, and hence the gradients of the triple velocity correlations make only a modest contribution to the overall budget of the Reynolds stresses. In such flows, defects in modelling the triple velocity correlations are easily concealed by shortcomings in the closure of the other unknowns in equation (7). In other flows, such as in the self-similar wake behind a bluff body, for example, the ratio of production to dissipation is only about 10 percent, with the remainder of the balance provided by the gradients of the triple correlations. It is precisely in these "weakly sheared" 
flows that current turbulence closures seem to fail very badly.

While the formulation of a triple-velocity correlations model that can be used in practical computations is outside the scope of this work, it is nevertheless useful to illustrate how such a model could be formulated. It is reasonable to argue that terms that are bilinear in $\left(\overline{u_{i} u_{j}}\right)_{, k}$ and $\overline{u_{i} u_{j}}$ should be included in the compact model for the triple correlations, simply in order to ensure that these quantities will vanish as either the Reynolds stresses themselves, or as their spatial gradients, go to zero. Dependence upon the gradients of mean velocity can be introduced through the retention of a term that is bilinear in the Reynolds stress gradients and the mean rate of strain tensor. The resulting model would then read

$$
\begin{aligned}
-\overline{u_{i} u_{j} u_{k}} & =\alpha_{1} \frac{k}{\epsilon}\left(\overline{u_{i} u_{p}} \frac{\partial \overline{u_{j} u_{k}}}{\partial x_{p}}+\overline{u_{j} u_{p}} \frac{\partial \overline{u_{k} u_{i}}}{\partial x_{p}}+\overline{u_{k} u_{p}} \frac{\partial \overline{u_{i} u_{j}}}{\partial x_{p}}\right) \\
& +\alpha_{2} \frac{k^{3}}{\epsilon^{2}}\left(S_{p i} \frac{\partial \overline{u_{j} u_{k}}}{\partial x_{p}}+S_{p j} \frac{\partial \overline{u_{k} u_{i}}}{\partial x_{p}}+S_{p k} \frac{\partial \overline{u_{i} u_{j}}}{\partial x_{p}}\right)
\end{aligned}
$$

Alternatively, an argument can be made to the effect that the contributions to the triple-velocity correlations arising from $S_{i j}$ and $W_{i j}$ are of the same order and, as such, can be combined. Thus, with the retention of a pair of terms that are bilinear in $\left(\overline{u_{i} u_{j}}\right)_{, k}$ and $S_{i j}$ or $W_{i j}$ there results

$$
\begin{aligned}
-\overline{u_{i} u_{j} u_{k}} & =\alpha_{1} \frac{k}{\epsilon}\left(\overline{u_{i} u_{p}} \frac{\partial \overline{u_{j} u_{k}}}{\partial x_{p}}+\overline{u_{j} u_{p}} \frac{\partial \overline{u_{k} u_{i}}}{\partial x_{p}}+\overline{u_{k} u_{p}} \frac{\partial \overline{u_{i} u_{j}}}{\partial x_{p}}\right) \\
& +\alpha_{2} \frac{k^{3}}{\epsilon^{2}}\left(\frac{\partial U_{p}}{\partial x_{i}} \frac{\partial \overline{u_{j} u_{k}}}{\partial x_{p}}+\frac{\partial U_{p}}{\partial x_{j}} \frac{\partial \overline{u_{k} u_{i}}}{\partial x_{p}}+\frac{\partial U_{p}}{\partial x_{k}} \frac{\partial \overline{u_{i} u_{j}}}{\partial x_{p}}\right)
\end{aligned}
$$

The illustrative models represented by equations (23) and (24) are, to our knowledge, the first rational, algebraic and explicit representations for the triple velocity correlations that are formulated from theoretical considerations and that allow for the explicit dependence of these correlations on the gradients of mean velocity. The coefficients $\alpha_{1}$ and $\alpha_{2}$ can be taken as constants and assigned numerical values by reference to the extensive data sets now available from Direct Numerical Simulations (e.g. Mansour et al. 1988).

\section{Closure}

The purpose of this paper was to advance a rational approach to modelling the triple velocity correlations of turbulence. A rational model was obtained by recourse to Group Representation Theory and by the development, specifically for the present purposes, of a representation for a symmetric third-order tensor in terms of both second- and third-order tensors, which are invariant under the full orthogonal group. Analysis of previous models showed all to be an incomplete subset of the full formulation that lacked, in particular, an explicit dependence on the gradients of mean velocity. This dependence appears explicitly in our representation and can therefore provide the basis for the development of a rational model for the triple velocity correlation. A demonstration of how this model may be formulated was provided. 


\title{
Appendix
}

\section{On the Representation of Third-Order Tensors}

\author{
G. F. Smith \\ Department of Mechanical Engineering 8 Mechanics \\ Lehigh University, Bethlehem, PA 18015, USA
}

The following notation is adopted in this appendix:

$$
\begin{aligned}
\tau_{p q} & \equiv \overline{u_{p} u_{q}} \\
A_{p q r} & \equiv\left(\overline{u_{p} u_{q}}\right)_{, r} \\
\mathcal{C}_{i j k} & \equiv \overline{u_{i} u_{j} u_{k}}
\end{aligned}
$$

We consider the problem of determining the form of a third-order symmetric tensorvalued polynomial function

$$
\mathcal{C}_{i j k}=\mathcal{F}_{i j k}\left(A_{p q r}, \tau_{p q}, S_{p q}, W_{p q}\right),
$$

which is invariant under the orthogonal group $O_{3}$ and which will be referred to as an isotropic function. The group $O_{3}$ is comprised of all $3 \times 3$ orthogonal matrices $\mathbf{Q}$ which satisfy

$$
\mathbf{Q Q}^{T}=\mathbf{Q}^{T} \mathbf{Q}=\mathbf{I}=\operatorname{diag}(1,1,1), \operatorname{det} \mathbf{Q}= \pm 1 .
$$

The tensors appearing in (A1) satisfy

$$
\begin{gathered}
\mathcal{C}_{i j k}=\mathcal{C}_{j k i}=\mathcal{C}_{k i j}=\mathcal{C}_{i k j}=\mathcal{C}_{k j i}=\mathcal{C}_{j i k}, \\
A_{i j k}=A_{j i k}, \quad \tau_{i j}=\tau_{j i}, \quad S_{i j}=S_{j i}, \quad W_{i j}=W_{j i} .
\end{gathered}
$$

We may readily determine the number $n_{1}, n_{2}, n_{3}, n_{4}$ of linearly independent thirdorder tensor-valued functions which are of degree $1,2,3,4$ respectively in the components of the $A_{i j k}, \ldots, W_{i j}$ and which are invariant under $O_{3}$. The procedure employed is similar to that adopted by Smith (1968). We have

$$
n_{1}=3, \quad n_{2}=27, \quad n_{3}=225, \quad n_{4}=1429 .
$$

The large number of terms of degree 3 and 4 in the $A_{i j k}, \ldots, W_{i j}$ leads us to limit consideration to the terms of degree $\leq 2$ appearing in (A1). Let

$$
B_{i j}^{(1)}=\tau_{i j}, \quad B_{i j}^{(2)}=S_{i j}, \quad B_{i j}^{(3)}=W_{i j} .
$$

The terms of degree $\leq 2$ appearing in the polynomial expression (A1) may then be written as

$$
\begin{aligned}
\mathcal{C}_{i j k}= & C_{i j k}+\sum_{\alpha=1}^{3} D_{i j k l m}^{(\alpha)} B_{l m}^{(\alpha)}+E_{i j k l m n} A_{l m n}+ \\
& +\sum_{\alpha, \beta=1 ; \alpha \leq \beta}^{3} F_{i j k l m n p}^{(\alpha, \beta)} B_{l m}^{(\alpha)} B_{n p}^{(\beta)}+\sum_{\alpha=1}^{3} G_{i j k l m n p q}^{(\alpha)} A_{l m n} B_{p q}^{(\alpha)}+ \\
& +H_{i j k l m n p q r} A_{l m n} A_{p q r} .
\end{aligned}
$$


The tensors $C_{i j k}, D_{i j k l m}^{(\alpha)}, \ldots, H_{i j k l m n p q r}$ appearing in (A6) are referred to as property tensors. The requirement that (A6) be invariant under $O_{3}$ imposes restrictions on the property tensors. Thus, the tensors $C_{i j k}, D_{i j k l m}^{(\alpha)}, \ldots$ must be invariant under $O_{3}$, i.e.,

$$
\begin{gathered}
C_{i j k}=Q_{i p} Q_{j q} Q_{k r} C_{p q r} \\
D_{i j k l m}^{(\alpha)}=Q_{i p} Q_{j q} Q_{k r} Q_{l s} Q_{m t} D_{p q r s t}^{(\alpha)}, \quad(\alpha=(1,2,3), \ldots
\end{gathered}
$$

must hold for all $\mathbf{Q}=\left\|Q_{i p}\right\|$ belonging to $O_{3}$. Such tensors are referred to as isotropic tensors. There are no tensors of odd order which are invariant under $O_{3}$. Even order tensors which are invariant under $O_{3}$ are expressible in terms of tensors of the form $\delta_{i j}, \delta_{i j} \delta_{k l}, \delta_{i j} \delta_{k l} \delta_{m n}, \ldots$ where $\delta_{i j}$ denotes the Kronecker delta tensor defined by

$$
\begin{gathered}
\delta_{i j}=1 \quad \text { if } i j=11,22,33 \\
\delta_{i j}=0 \quad \text { if } i j=12,21,13,31,23,32 .
\end{gathered}
$$

See, for example, Smith (1994, p. 94) or Smith (1968). Thus, the tensors of odd order $C_{i j k}, D_{i j k l m}^{(\alpha)}, F_{i j k l m n p}^{(\alpha, \beta)}, H_{i j k l m n p q r}$ appearing in (A6) are null tensors, i.e., all components of these tensors are zero. The expression (A6) then reduces to

$$
\begin{aligned}
\mathcal{C}_{i j k}= & E_{i j k l m n} A_{l m n}+G_{i j k l m n p q}^{(1)} A_{l m n} \tau_{p q}+ \\
& +G_{i j k l m n p q}^{(2)} A_{l m n} S_{p q}+G_{i j k l m n p q}^{(3)} A_{l m n} W_{p q} .
\end{aligned}
$$

We may readily show that the number of linearly independent third-order symmetric tensor-valued isotropic functions which are linear in $A_{i j k}$, bilinear in $A_{i j k}$ and $\tau_{i j}$, bilinear in $A_{i j k}$ and $S_{i j}$, and bilinear in $A_{i j k}$ and $W_{i j}$ are given by $3,11,11$ and 5 respectively. We shall employ the notation $\sum B_{i \ldots j \ldots k}$ to indicate the sum of the three tensors obtained by cyclic permutation of the subscripts $i j k$ on the summand. For example,

$$
\begin{gathered}
\sum A_{i j k}=A_{i j k}+A_{j k i}+A_{k i j}, \\
\sum A_{i j p} \tau_{p k}=A_{i j p} \tau_{p k}+A_{j k p} \tau_{p i}+A_{k i p} \tau_{p j} .
\end{gathered}
$$

The three linearly independent terms appearing in (A9) which are linear in $A_{i j k}$ are given by

$$
\sum A_{i j k}, \sum A_{i p p} \delta_{j k}, \sum A_{p p i} \delta_{j k}
$$

The eleven linearly independent terms appearing in (A9) which are bilinear in $A_{i j k}$ and $\tau_{i j}$ are given by

$$
\begin{aligned}
\tau_{p p} \sum A_{i j k}, & \sum A_{i p p} \tau_{j k}, \sum A_{p p i} \tau_{j k}, \sum A_{i j p} \tau_{p k}, \sum\left(A_{i p j}+A_{j p i}\right) \tau_{p k} \\
& \sum A_{i p q} \tau_{p q} \delta_{j k}, \sum A_{p q i} \tau_{p q} \delta_{j k}, \sum A_{p p q} \tau_{q i} \delta_{j k}, \\
& \sum A_{q p p} \tau_{q i} \delta_{j k}, \tau_{q q} \sum A_{p p i} \delta_{j k}, \tau_{q q} \sum A_{i p p} \delta_{j k} .
\end{aligned}
$$


The eleven linearly independent terms appearing in (A9) which are bilinear in $A_{i j k}$ and $S_{i j}$ are given by

$$
\begin{aligned}
S_{p p} \sum A_{i j k}, & \sum A_{i p p} S_{j k}, \sum A_{p p i} S_{j k}, \sum A_{i j p} S_{p k}, \sum\left(A_{i p j}+A_{j p i}\right) S_{p k} \\
& \sum A_{i p q} S_{p q} \delta_{j k}, \sum A_{p q i} S_{p q} \delta_{j k}, \sum A_{p p q} S_{q i} \delta_{j k}, \\
& \sum A_{q p p} S_{q i} \delta_{j k}, S_{q q} \sum A_{p p i} \delta_{j k}, S_{q q} \sum A_{i p p} \delta_{j k} .
\end{aligned}
$$

The five linearly independent terms appearing in (A9) which are bilinear in $A_{i j k}$ and $W_{i j}$ are given by

$$
\begin{gathered}
\sum A_{i j p} W_{p k}, \sum\left(A_{i p j}+A_{j p i}\right) W_{p k}, \sum A_{i p q} W_{p q} \delta_{j k} \\
\sum A_{p p q} W_{q i} \delta_{j k}, \sum A_{q p p} S_{q i} \delta_{j k} .
\end{gathered}
$$

The polynomial expression (A1), which is invariant under $O_{3}$ and in which terms of degree three or greater in the $A_{i j k}, \ldots, W_{i j}$ are neglected, is then given as a linear combination of the 30 terms listed above, i.e.,

$$
\mathcal{C}_{i j k}=\sum_{\gamma=1}^{30} c_{\gamma} \Phi_{i j k}^{(\gamma)}
$$

where the $\Phi_{i j k}^{(\gamma)}(\gamma=1, \ldots, 30)$ are the third-order symmetric tensor-valued isotropic functions listed in (A11),...,(A14). The $c_{\gamma}$ are material constants. 


\section{References}

Chou, P. Y. 1945: On Velocity Correlations and the Solutions of the Equations of Turbulent Fluctuation. Quart. Appl. Maths., Vol. 3, pp. 38-54.

Cormack, D. E.; Leal, L. G.; and Seinfeld, J. H. 1978: An Evaluation of Mean Reynolds Stress Turbulence Models: The Triple Velocity Correlation. Trans. ASME J. Fluid Engrg., Vol. 100, pp. 47-54.

Daly, B. J.; and Harlow, F. H. 1970: Transport Equations of Turbulence. Phys. Fluids, Vol. 13, pp. 2634-2649.

Hanjalic, K.; and Launder, B. E. 1972: A Reynolds Stress Model of Turbulence and Its Application to Thin Shear Flows. J. Fluid Mech., Vol. 52, pp. 609-638.

Hinze, J. O. 1975: Turbulence, 2nd ed. McGraw-Hill.

Lumley, J. L. 1978: Computational Modeling of Turbulent Flows. Advances in Applied Mechanics, C-S. Yih, ed. Academic Press, Vol. 18, pp. 123-176.

Magnaudet, J. 1993: Modelling of Inhomogeneous Turbulence in the Absence of Mean Velocity Gradients. Appl. Sci. Res., Vol. 51, pp. 525-531.

Mansour, N. N.; Kim, J.; and Moin, P. 1988: Reynolds-Stress and DissipationRate Budgets in a Turbulent Channel Flow. J. Fluid Mech., Vol. 194, pp. 15-44.

Mellor, G. L.: and Herring, H. J. 1973: A Survey of the Mean Turbulent Field Closure Models. AIAA J., Vol. 11, pp. 590-599.

Millionshtchikov, M. D.: 1941: On the Theory of Homogeneous Isotropic Turbulence. C. R. Acad. Sci. S.S.S.R., Vol. 32, pp. 615-619.

Pennisi, S.: 1992: On Third Order Tensor-Valued Isotropic Functions. Int. J. Engrg. Sci., Vol. 30, pp. 679-692.

Schwarz, W. R.: and Bradshaw, P. 1994: Term-By-Term Tests of Stress-Transport Turbulence Models in a Three-Dimensional Boundary Layer. Phys. Fluids, Vol. 6, pp. $986-998$.

Shir, C. C. 1973: A Preliminary Numerical Study of Atmospheric Turbulent Flows in the Idealized Planetary Boundary Layer. J. Atmos. Sci., Vol. 30, pp. 1327-1339.

Smith, G. F. 1965: On Isotropic Integrity Bases. Arch. Rational Mech. Anal., Vol. 18, pp. 282-292.

Smith, G. F. 1968: Isotropic Tensors and Rotation Tensors of Dimension $m$ and Order $n$. Tensor, Vol. 19, pp. 79-88.

Smith, G. F. 1994: Constitutive Equations for Anisotropic and Isotropic Materials. North-Holland.

Speziale, C. G. 1991: Analytical Methods for the Development of Reynolds Stress Closures in Turbulence. Annu. Rev. Fluid Mech. Vol. 23, pp. 107-157. 
Public reporting burden for this collection of information is estimated to average 1 hour per response, including the time for reviewing instructions, searching existing data sources, gathering and maintaining the data needed, and completing and reviewing the collection of information. Send comments regarding this burden estimate or any other aspect of this collection of information, including suggestions for reducing this burden, to Washington Headquarters Services, Directorate for Information Operations and Reports, 1215 Jefferson Davis Highway, Suite 1204, Arlington, VA 22202-4302, and to the Office of Management and Budget, Paperwork Reduction Project (0704-0188), Washington, DC 20503.

\begin{tabular}{|l|l}
\hline 1. AGENCY USE ONLY (Leave blank) & $\begin{array}{c}\text { 2. REPORT DATE } \\
\text { April } 1999\end{array}$ \\
\hline
\end{tabular}

4. TITLE AND SUBTITLE

Towards a Rational Model for the Triple Velocity Correlations of Turbulence

6. AUTHOR(S)

B. A. Younis, T. B. Gatski, and C. G. Speziale

\section{REPORT TYPE AND DATES COVERED}

Technical Memorandum

\begin{tabular}{l|l}
\hline 7. PERFORMING ORGANIZATION NAME(S) AND ADDRESS(ES) & \\
NASA Langley Research Center & $\begin{array}{l}\text { 8. PERFORMING ORGANIZATION } \\
\text { REPORT NUMBER } \\
\text { L-17847 } \\
\text { Hampton, VA 23681-2199 }\end{array}$ \\
& \\
\hline $\begin{array}{l}\text { 9. SPONSORING/MONITORING AGENCY NAME(S) AND ADDRESS(ES) } \\
\text { Washional Aeronautics and Space Administration }\end{array}$ & $\begin{array}{l}\text { 10. SPONSORING/MONITORING } \\
\text { AGENCY REPORT NUMBER } \\
\text { NASA/TM-1999-209134 }\end{array}$ \\
& \\
& \\
\hline
\end{tabular}

11. SUPPLEMENTARY NOTES

\author{
12a. DISTRIBUTION/AVAILABILITY STATEMENT \\ Unclassified-Unlimited \\ Subject Category 34 \\ Availability: NASA CASI (301) 621-0390
}

\section{NUMB \\ 522-31-61-01}

